\title{
Exercise in Adolescent Depression: Fitness, Clinical Outcomes, and BDNF
}

\author{
Richard Dopp ${ }^{1 *}$, Zachary Miklja², Emily Jurries ${ }^{3}$, and Ann Mooney ${ }^{1}$ \\ ${ }^{1}$ Department of Psychiatry, University of Michigan, Ann Arbor, MI, USA \\ ${ }^{2}$ Department of Neurosurgery, University of Michigan, Ann Arbor, MI, USA \\ ${ }^{3} \mathrm{~A}$ Little Counseling, PLLC, Wake Forest, NC, USA
}

*Corresponding author: Richard Dopp, Department of Psychiatry, University of Michigan, Ann Arbor, MI, USA, Tel: 734-945-6124; 734-9368907; E-mail: dopp@med.umich.edu

Received: 21 Apr, 2020 | Accepted: 08 May, 2020 | Published: 15 May, 2020

Citation: Dopp R, Miklja Z, Jurries E, Mooney A (2020) Exercise in Adolescent Depression: Fitness, Clinical Outcomes, and BDNF. J Neurol Neurobiol 6(3): dx.doi.org/10.16966/2379-7150.167

Copyright: (C) Dopp R, et al. This is an open-access article distributed under the terms of the Creative Commons Attribution License, which permits unrestricted use, distribution, and reproduction in any medium, provided the original author and source are credited.

\section{Abstract}

Introduction: Despite the initiation of treatment for depression including medications and evidence-based psychotherapies, many adolescents continue to have depressive symptoms. A 2017 meta-analysis of exercise research for this population summarized that physical activity appears to improve depression symptoms in adolescents, but the need for larger trials was emphasized. Most importantly, the physiological and neurological mechanisms of action through which exercise exerts its antidepressant effects must be explored.

Objectives: The objective of this study was to assess the feasibility of a group exercise intervention for adolescents with depressive disorders. To investigate physiologic changes, serum biomarkers were examined including brain-derived neurotrophic factor (BDNF). Secondary analyses explored relationships among depressive symptoms, exercise self-efficacy, and fitness.

Methods: Adolescents with depression (Children's Depression Rating Scale-Revised, CDRS-R $\geq 40$ ) participated in a group intervention for 12 weeks of aerobic exercise (1X in group and $2 X$ independently each week). Blood draws were taken pre- and post-intervention. At weeks 1 and 12 , the Balke Fitness Test was administered measuring exertion ratings and heart rates during treadmill activity.

Results: Participants had a significant decrease in depressive symptoms over the 12-week intervention. Mean CDRS-R score of completers was 52.2 at baseline and 29.6 post-intervention, for a decrease of 22.5 points. Paired samples $t$-tests showed that the decrease in CDRS-R scores from baseline to week 12 was statistically significant $[t(12)=9.12, p<.001]$. There was a significant increase in plasma BDNF between baseline and post-intervention for the completers of the exercise intervention $[t(12)=-2.6, p<.03]$. Reductions in mean exertion ratings on the Balke Fitness Test from the final minute of testing at weeks 1 and 12 were significantly correlated with reductions in CDRS-R scores $(r=.57, p=.04)$.

Conclusions: The significant decrease in depressive symptoms over the 12-week intervention suggests that exercise is effective in the treatment of depression in adolescents. When comparing data from these adolescents with depressive disorders, there were significant reductions in depression and significant increases in BDNF after 12 weeks of exercise.

Keywords: Exercise; Adolescent; Depression; Suicide ideation; Brain-derived Neurotrophic Factor (BDNF)

\section{Introduction}

Data from Monitoring the Future study demonstrates significant increases in reports of depressive symptoms among adolescents between 1991 and 2018, consistent with increases in depressive disorders and suicides [1]. There is convincing evidence to suggest that serum levels of brain-derived neurotrophic factor (BDNF) are lower for individuals suffering from major depressive disorder [2]. Abnormalities in BDNF levels and in the BDNF signaling pathway may be contributors to suicidal thoughts and behaviors [3].

At the same time, numerous studies suggest that serum levels of $\mathrm{BDNF}$ often normalize in response to treatment with antidepressant medications [2]. Unfortunately, many adolescents with depression never seek medical treatment. Even for adolescents taking antidepressant medications in combination with cognitive behavioral therapy, nearly two thirds fail to achieve remission of their depression [4].

Physical activity has been shown to treat depression in adults [5] and has been demonstrated as a feasible treatment for adolescents with depression [6]. A 2017 meta-analysis of exercise research for this population summarized that exercise and physical activity appear to improve depression symptoms in adolescents, especially in clinical samples, suggesting that exercise may indeed be a useful treatment strategy for adolescents with depression [7]. 
Exercise is also known to increase BDNF levels in the brain, confirmed by the measurement of simultaneous blood samples obtained from the radial artery and the internal jugular vein as human subjects were actively exercising [8,9]. Rodent studies have demonstrated that exercise induces BDNF activation, both peripherally in skeletal muscle and centrally in the hippocampus, via similar pathways [10]. Increases in BDNF appear to be one of the mechanisms through which exercise exerts its antidepressant effects.

\section{Objectives}

The objective was to assess feasibility and effectiveness of a group exercise intervention for adolescents with depressive disorders. Secondary analyses explored relationships among depressive symptoms, exercise self-efficacy, and fitness. To investigate physiologic changes, serum biomarkers were examined pre- and post-intervention, including BDNF. We hypothesized that participation in this 12-week exercise intervention would be associated with significant decreases in symptoms of depression and with increases in measured levels of BDNF.

\section{Methods}

Adolescents with depression and low activity levels were consented and accepted into a group exercise intervention for 12 weeks of aerobic exercise (1X in group and 2X independently each week). Blood draws were taken pre- and post-intervention to assess changes in metabolic biomarkers. Participant information is included in Table 1.

\section{Clinical Measures}

Children's Depression Rating Scale, Revised (CDRS-R); [11] is a semi-structured interview assessing depressive symptoms. CDRS-R was administered at baseline and post-intervention.

Balke Fitness Test is a 6-minute treadmill task with increasing incline and a 6-20 exertion rating [12]. The Balke Protocol is designed to provide a measure of cardiovascular fitness. Stage 1 of Balke Fitness Test is recorded after 2 minutes; 1 minute at $6 \%$ incline and 1 minute at $8 \%$ incline with a speed of $3 \mathrm{mph}$. Stage 5 of Balke Fitness Test is recorded after 6 minutes of increasing incline at $3 \mathrm{mph}$. Participant reaches maximum incline of $12 \%$ at the fourth minute. This fitness test was conducted at weeks 1 and 12 .

Exercise Self-Efficacy Scale (ESES) [13] is an 18-item questionnaire that assessed the extent to which one believes he/she can exercise under certain circumstances.

Suicidal Ideation Questionnaire Junior (SIQ-JR) [14] is a 15item assessment (6 points maximum per question) for how often an adolescent is contemplating suicide. A score of 23 out of 90 is a cutoff used in establishing the risk for suicide.
Biological Markers Serum blood draws (baseline and postintervention) assessing BDNF.

\section{Results}

Mean CDRS-R score of completers was 52.2 at baseline and 29.6 post-intervention, for a decrease of 22.5 points. Paired samples t-tests showed that the decrease in CDRS-R scores from baseline to week 12 was statistically significant $[t(12)=9.12, p<.001]$.

When comparing baseline and week 12 values on the Balke Fitness Test, mean exertion scores were significantly reduced for both Stage 1 of testing $[\mathrm{t}(12)=2.3, \mathrm{p}<.04)$ and Stage 5 of testing $[\mathrm{t}(12)=2.3, \mathrm{p}<.04]$, as seen in figure 1. Exertion ratings from the final minute of the Balke Fitness Test were negatively correlated with Exercise Self-Efficacy Scores at baseline $(\mathrm{r}=-.53, \mathrm{p}<.02)$ and at week $12(\mathrm{r}=-.82, \mathrm{p}=.001)$.

Reductions in mean exertion ratings from the final minute of testing were significantly correlated with reductions in CDRS-R scores $(\mathrm{r}=.57, \mathrm{p}=.04)$, as seen in figure 2 , and SIQ scores $(\mathrm{r}=.59, \mathrm{p}<0.04)$, as seen in figure 3.

There was a significant increase in plasma BDNF between baseline and post-intervention for the completers of the exercise intervention $[t(12)=-2.6, p<.03]$, as seen in figure 4 .

\section{Limitations}

This was a feasibility study to see if group exercise would have the same impact on depression levels for adolescents as has been established for those in individual exercise [6]. There was no control condition in this study which required the reliance on within-subjects comparisons in our analyses. The small sample size was a limiting variable. While the dropout rate was indicative of the challenges of engaging adolescents with depression in an exercise regimen, it further reduced the sample size.

Concurrent treatment may have been a confounding variable. Research participants were allowed to continue their current treatment while they were in this study. If they were on medications, they were expected to be on a stable dose for 4-6 weeks prior to initiation of the exercise intervention. Of the 13 completers, all were either on medications, in psychotherapy, or both. The impact of these treatments occurring simultaneously with the exercise regimen is thus unknown.

\section{Discussion}

Exercise should be considered as part of clinical recommendations for all youth and adults with depressive disorders. Research in rodents has demonstrated the important role of BDNF in the molecular mechanisms fostering neuronal plasticity [15]. More recent research has shown that myokines, muscle-derived molecules affecting aspects

Table 1: Participant information.

\begin{tabular}{|c|c|c|c|}
\hline & Completers(13) & Non-Completers(12) & Total(25) \\
\hline \multicolumn{4}{|l|}{ Sex } \\
\hline Females & 7 & 9 & 16 \\
\hline Males & 6 & 3 & 9 \\
\hline Age in years & $14.4(2.2)$ & $15.5(1.8)$ & $14.9(2.1)$ \\
\hline \multicolumn{4}{|l|}{ Diagnosis } \\
\hline MDD & 2 & 3 & 5 \\
\hline Depressive disorder NOS & 10 & 6 & 16 \\
\hline Bipolar disorder & 1 & 3 & 4 \\
\hline Concurrent use of medications & 9 & 12 & 21 \\
\hline
\end{tabular}

Citation: Dopp R, Miklja Z, Jurries E, Mooney A (2020) Exercise in Adolescent Depression: Fitness, Clinical Outcomes, and BDNF. J Neurol 


\section{Balke Exertion Rates in Stage 1 and Stage \\ 5 at Week 1 and Week 12}
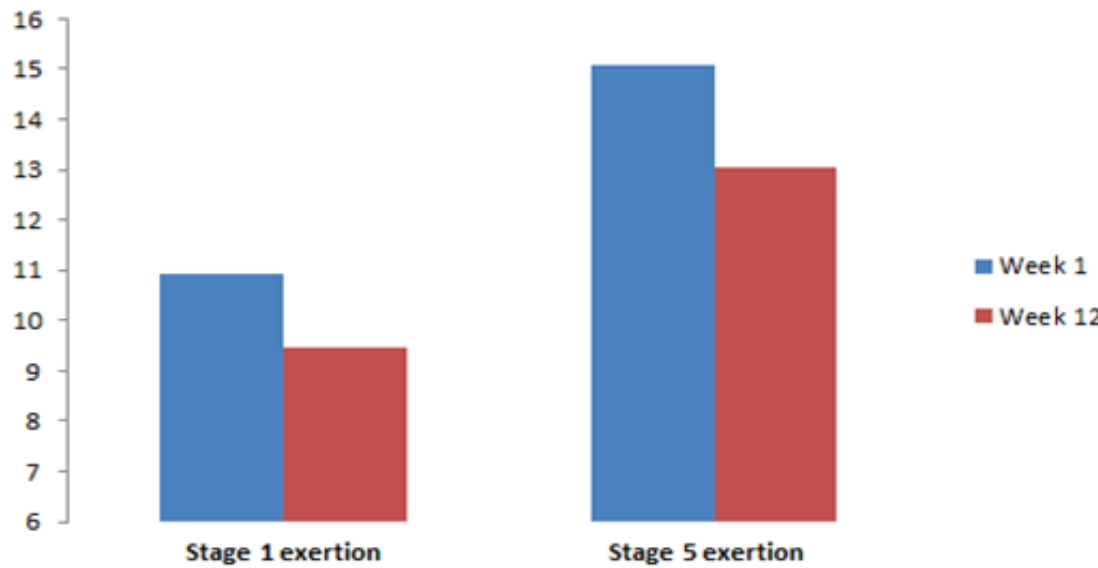

a week 12

Stage 5 exertion

Figure 1: Reductions in ratings of perceived exertion (RPE) with ongoing exercise participation.

\section{Correlation between Change in Stage 5 Exertion Rating and Change in CDRS} scores

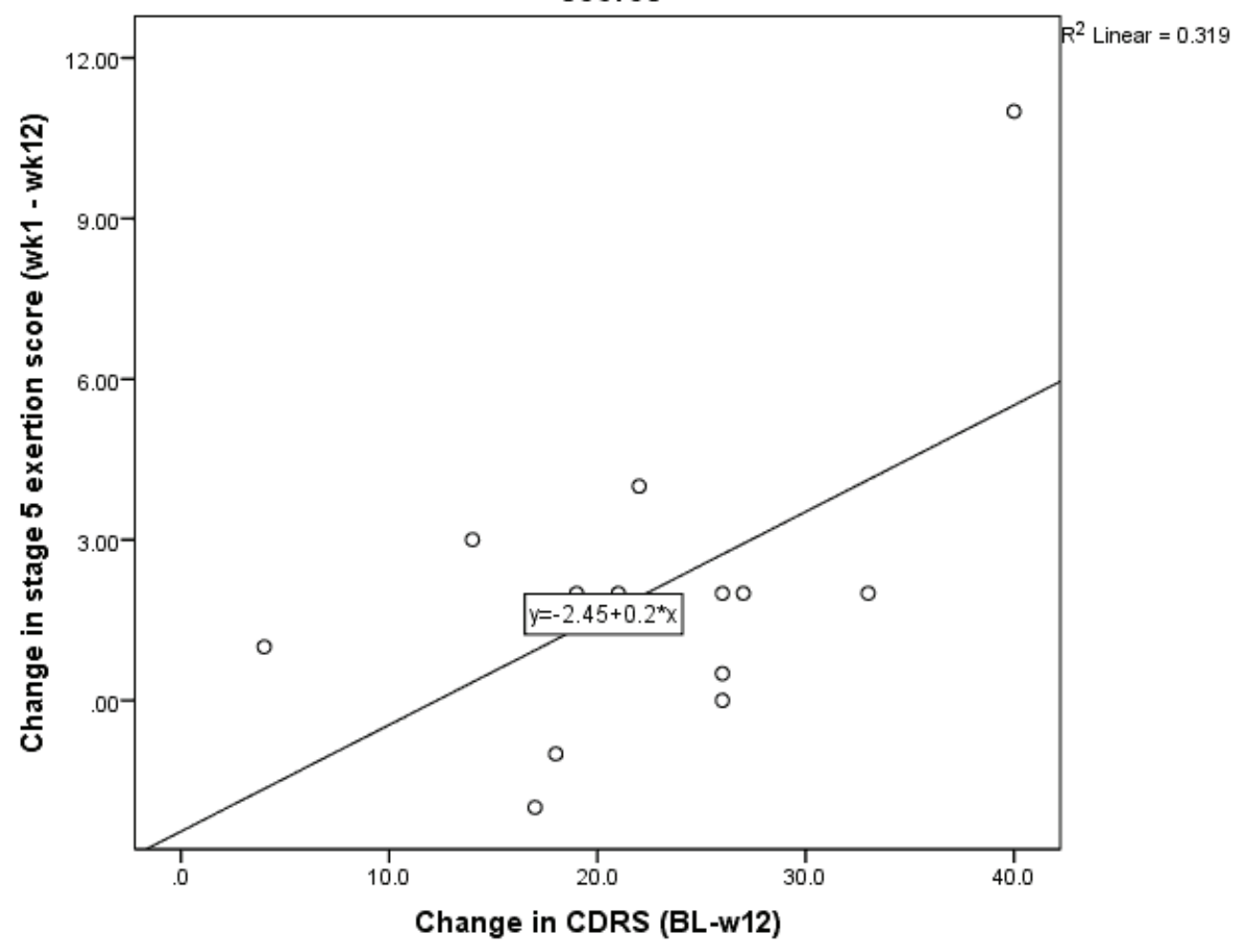

Figure 2: Reductions in depression severity were correlated with reductions in RPE. 


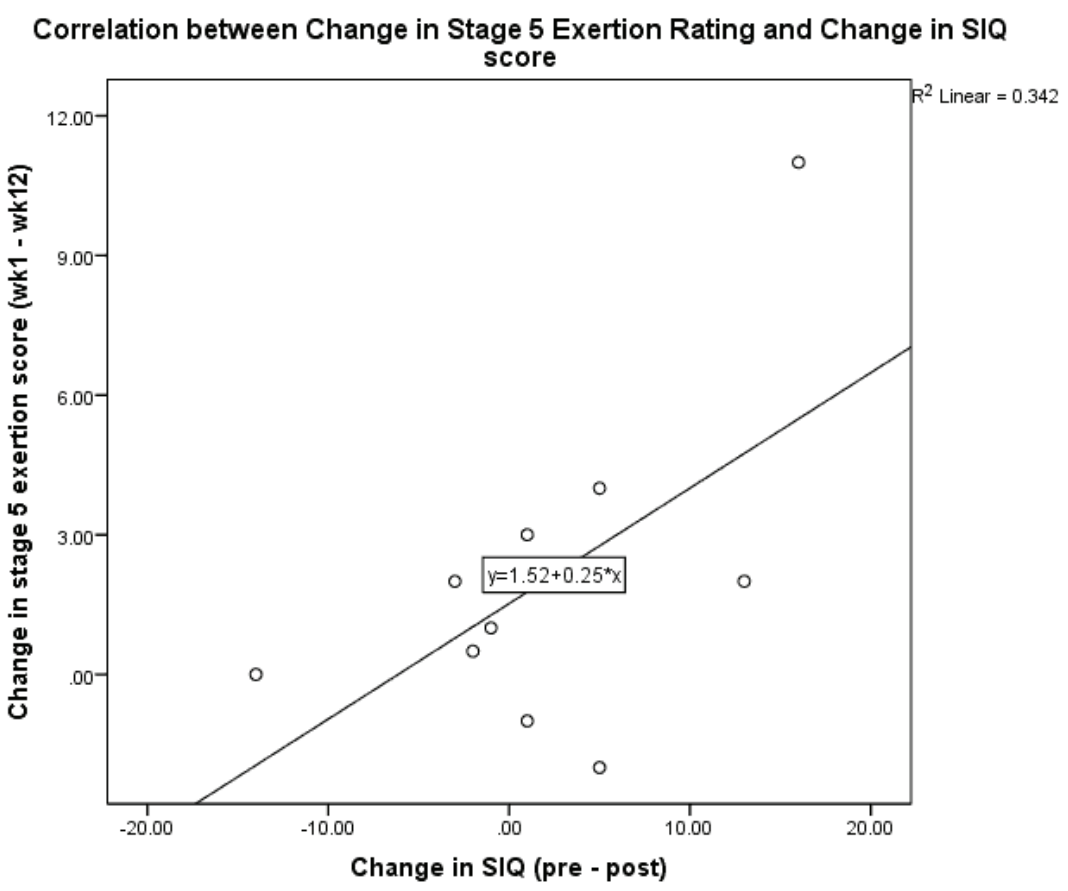

Figure 3: Reductions in severity of suicidal ideation correlated with reductions in RPE.

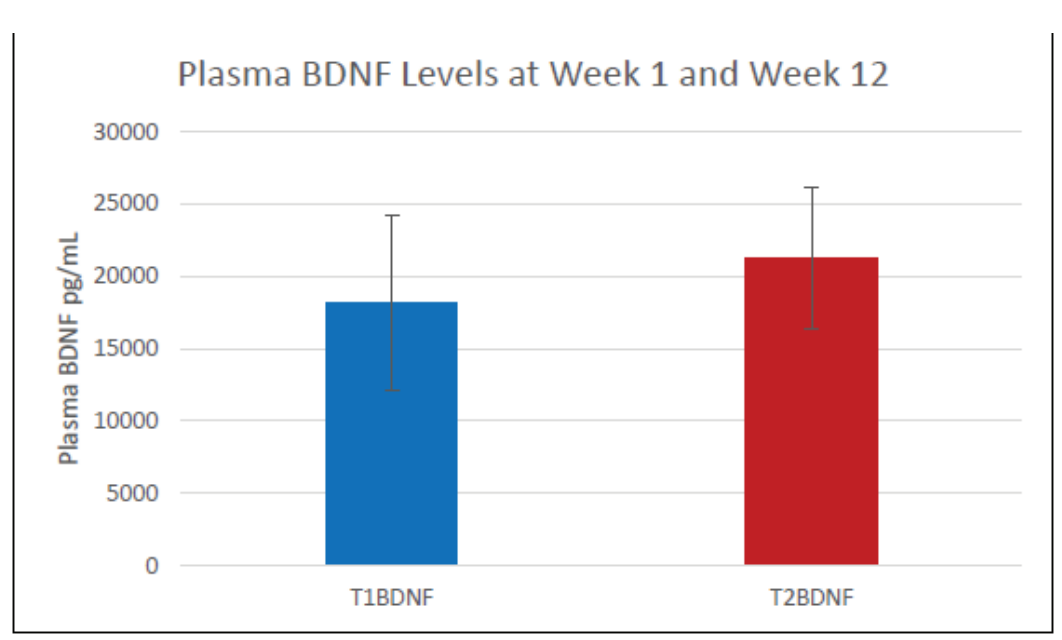

Figure 4: BDNF levels were significantly increased from week 1 (T1) to week 12 (T2).

of metabolism, may serve as "exercise factors" that may contribute to improved brain health [16]. Wrann and colleagues identified similar mechanisms of BDNF induction in both skeletal muscle and in the hippocampus via a PGC-1a/FNDC5 pathway [17]. Peripheral FNDC5 is cleaved resulting in a product called irisin which enhances BDNF gene expression in the hippocampus, a major site of activation and brain changes following exercise $[10,17]$.

In this intervention, improved fitness, as measured by significant decreases in ratings of physical exertion, predicted reductions in depression and suicidal ideation. Among these adolescents with depression who completed the 12-week exercise intervention, a significant increase in BDNF was observed between baseline and postintervention.

\section{Conclusions}

Exercise is an effective treatment for depression among adolescents. BDNF plays a key role in neuroplasticity of the brain and its ability to adapt and respond to new challenges. Increases in BDNF are associated with improved brain plasticity and cognition. Similar to the increases in BDNF reported with other antidepressant treatments [18], reductions in depression in these adolescents following exercise may be a result of increases in BDNF. The results of this research project suggest that adolescents with depressive disorders can use 
exercise as part of their treatment to effectively reduce their symptoms of depression, increasing BDNF levels that improve brain health and promote synaptic plasticity.

Exercise should be considered as part of treatment for adolescents with clinically significant depressive disorders.

\section{Acknowledgements}

This research project was funded by the University of Michigan Depression Center's Executive Director's Innovation Fund, the Rachel Upjohn Clinical Scholars award, the Feldman Award, and by Grant Number P30DK020572 (MDRC) from the National Institute of Diabetes and Digestive and Kidney Diseases. We appreciate the BDNF analytic assistance from Jason Whalen and the Michigan Diabetes Research Center Laboratory and the clinical support from the Michigan Institute for Clinical and Health Research (grant numbers: UL1TR000433 and UL1TR002240).

\section{References}

1. Keyes KM, Gary D, O'Malley PM, Hamilton A, Schulenberg J (2019) Recent increases in depressive symptoms among US adolescents: trends from 1991 to 2018. Soc Psychiatry Psychiatr Epidemiol 54: 987-996.

2. Sen S, Duman R, Sanacora G (2008) Serum brain-derived neurotrophic factor, depression, and antidepressant medications: meta-analyses and implications. Biol Psychiatry 64: 527-532.

3. Dwivedi Y (2009) Brain-derived neurotrophic factor: role in depression and suicide. Neuropsychiatr Dis Treat 5: 433-449.

4. Kennard B, Silva S, Vitiello B, Curry J, Kratochvil C, et al. (2006) Remission and residual symptoms after short-term treatment in the Treatment of Adolescents with Depression Study (TADS). J Am Acad Child Adolesc Psychiatry 45: 1404-1411.

5. Dunn AL, Trivedi MH, Kampert JB, Clark CG, Chambliss HO (2002) The DOSE study: a clinical trial to examine efficacy and dose response of exercise as treatment for depression. Control Clin Trials 23: 584-603.

6. Dopp RR, Mooney AJ, Armitage R, King C (2012) Exercise for Adolescents with Depressive Disorders: A Feasibility Study. Depress Res Treat 2012.

7. Carter T, Morres I, Meade O, Patrick C (2017) Exercise for depression in adolescents: A meta-analysis of randomised controlled trials. Eur Psychiatry 41: S433.

8. Rasmussen P, Brassard P, Adser H, Pedersen MV, Leick L, et al. (2009) Evidence for a release of brain-derived neurotrophic factor from the brain during exercise. Exp Physiol 94: 1062-1069.

9. Seifert $T$, Brassard $P$, Wissenberg $M$, Rasmussen $P$, Nordby $P$, et al. (2010) Endurance training enhances BDNF release from the human brain. Am J Physiol Regul Integr Comp Physiol 298: R372-R377.

10. Wrann CD, White JP, Salogiannnis J, Laznik-Bogoslavski D, Wu J, et al. (2013) Exercise induces hippocampal BDNF through a PGC-1 $\alpha$ / FNDC5 pathway. Cell Metab 8: 649-659.

11. Mayes TL, Bernstein IH, Haley CL, Kennard BD, Emslie GJ (2010) Psychometric properties of the Children's Depression Rating ScaleRevised in adolescents. J Child Adolesc Psychopharmacol 20: 513516

12. Marinov B, Kostianev S, Turnovska T (2003) Modified treadmill protocol for evaluation of physical fitness in pediatric age group-comparison with Bruce and Balke protocols. Acta Physiol Pharmacol Bulg 27: 47-51.

13. Kroll T, Kehn M, Ho PS, Groah S (2007) The SCI Exercise Self-Efficacy Scale (ESES): development and psychometric properties. Int J Behav Nutr Phys Act 4: 34.

14. Reynolds WM, Mazza JM (1999) Assessment of Suicidal Ideation in Inner-City Children and Young Adolescents: Reliability and Validity of the Suicidal Ideation Questionnaire-JR. School Psychol Rev 28: 17-30.

15. Gómez-Pinilla F, Ying Z, Roy RR, Molteni R, Edgerton VR (2002) Voluntary exercise induces a BDNF-mediated mechanism that promotes neuroplasticity. J Neurophysiol 88: 2187-2195.

16. Kim S, Choi JY, Moon S, Park DH, Kwak HB, et al. (2019) Roles of myokines in exercise-induced improvement of neuropsychiatric function. Pflugers Arch 471: 491-505.

17. Xu B (2013) BDNF (I)rising from exercise. Cell Metab 18: 612-614.

18. Björkholm C, Monteggia LM (2016) BDNF - a key transducer of antidepressant effects. Neuropharmacology 102: 72-79. 